\title{
Carotenoids and Retinoids
}

The Effect of Astaxanthin and Lutein on the Activation of Hepatic Stellate Cells and the Underlying Mechanisms (E01-01)

Minkyung Bae, Mi-Bo Kim, and Ji-Young Lee

University of Connecticut

Objective: Hepatic stellate cells (HSCs) play essential roles in the development of liver fibrosis by increasing accumulation of extracellular matrix proteins in the damaged liver. We previously demonstrated that astaxanthin (ASTX) and lutein (LT) have antifibrogenic properties in LX-2, a human HSC cell line, when testing 5 carotenoids, ASTX, LT, lycopene, zeaxanthin, and canthaxanthin. The objective of this study was to determine the mechanisms of the antifibrogenic effect of ASTX and LT in HSCs.

Methods: Primary HSCs isolated from C57BL/6J mice were cultured on uncoated plastic dishes for spontaneous activation in the presence or absence of $25 \mu \mathrm{M}$ of ASTX or LT to determine their effect on HSC activation. The cells were harvested at day 1 or day 7 for quiescent or activated HSCs, respectively, to measure the expression of fibrogenic genes. In addition, we determined whether the SMAD3 pathway mediates the antifibrogenic effect of ASTX and LT by measuring levels of phosphorylated SMAD3 and total SMAD3 in LX-2 cells stimulated by transforming growth factor $\beta 1$ in the presence or absence of ASTX and LT.

Results: Activation of mouse primary HSCs was confirmed by drastic induction of fibrogenic genes, such as $\alpha$-smooth muscle actin ( $\alpha$-SMA), procollagen type I $\alpha 1$ (Col1a1), Col3a1, Col6a1, and Col6a3, in activated HSCs compared with quiescent HSCs. Importantly, the presence of ASTX during HSC activation significantly reduced mRNA levels of $\alpha$-SMA, Col1a1, Col3a1, Col6a1, and Col6a3. Although LT also decreased the fibrogenic gene expression when it was present during HSC activation, the reduction was significantly less than that by ASTX. Furthermore, in LX-2 cells, transforming growth factor $\beta 1$ induced phosphorylation of SMAD3 by $>3$-fold. However, ASTX markedly reduced phosphorylation of SMAD3 by $\sim 40 \%$, whereas LT only decreased it by $\sim 10 \%$.

Conclusions: Although both ASTX and LT inhibited the activation of quiescent HSCs and decreased fibrogenic gene expression in HSCs, ASTX was more potent than LT in in terms of antifibrogenic effect, which was mediated by inhibition of SMAD3 phosphorylation. However, LT exerts an antifibrogenic action independent of the SMAD3 pathway.

Funding Sources

NIH 1R01DK108254-01, USDA Hatch CONS00972.

Fucoxanthin Inhibits Transforming Growth Factor $\beta 1$-induced Profibrogenic Gene Expression by Repressing SMAD3 in Hepatic Stellate Cells (E01-02)

Mi-Bo Kim, Minkyung Bae, Siqi Hu, Young-Ki Park, and Ji-Young Lee

University of Connecticut

Objective: Nonalcoholic steatohepatitis (NASH) is characterized by excessive fat accumulation, inflammation, and fibrosis in the liver. The activation of hepatic stellate cells (HSCs) is a critical event in the development of liver fibrosis as these cells are primarily responsible for the accumulation of extracellular matrix (ECM) in the injured liver. The objective of this study was to evaluate whether fucoxanthin (FCX), a carotenoid present in edible brown seaweeds, can inhibit fibrogenesis in LX-2 cells, a human HSC cell line, as well as primary mouse and human HSCs.

Methods: We measured the cytotoxicity of FCX in LX-2 cells. The effects of FCX on transforming growth factor $\beta 1$ (TGF $\beta 1$ )-induced profibrogenic gene expression and on the

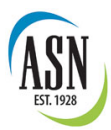



(c) 2018 American Society for Nutrition. All rights reserved.

Manuscript received September 20, 2017. Initial review completed March 23, 2018. Revision accepted May 27, 2018. Published online June 28, 2018. 
SMAD3 pathway were determined in LX-2 cells and human primary HSCs from quantitative real-time polymerase chain reaction and Western blot analysis. The modulatory roles of FCX in the activation of quiescent HSCs to activated HSCs were also evaluated in mouse primary HSCs isolated from C57BL/6J mice.

Results: FCX exhibited cell viability of $>90 \%$ and $>75 \%$ at concentrations of 2.5 and $5 \mu \mathrm{M}$, respectively. TGF $\beta 1$ increased the mRNA levels of profibrogenic genes, such as $\alpha$-smooth muscle actin ( $\alpha$-SMA) and procollagen type I $\alpha 1$ (Col1A1), in LX- 2 cells and human HSCs. However, FCX significantly inhibited the induction. As the SMAD3 pathway is known to mediate TGF $\beta 1$-induced fibrogenesis in HSCs, we determined whether FCX can regulate SMAD3 activation, i.e., phosphorylation, in LX-2 cells. TGF $\beta 1$ stimulated phosphorylation of SMAD3, which was markedly attenuated by FCX. When quiescent mouse primary cells were cultured on an uncoated plastic plate for activation for $4 \mathrm{~d}$, mRNA levels of $\alpha$-SMA, procollagen genes such as Col1a1, Col3a1, Col6a1, and Col6a3, and the tissue inhibitor metalloproteinase-1 were induced. However, the presence of FCX during the activation significantly reduced these mRNA levels. In addition, cells treated with FCX displayed more cytoplasmic lipid droplets, a characteristic of quiescent HSCs, than control cells.

Conclusion: The results suggest that FCX exerts antifibrogenic effects by preventing TGF $\beta 1$-induced profibrogenic gene expression, which it achieves by inhibiting SMAD3 activation in HSCs and by inhibiting the activation of quiescent HSCs.

\section{Funding Sources}

USDA Hatch CONS00972; USDA Multi-State Hatch CONS00916.

\section{$\beta$-Cryptoxanthin Alleviates High-Refined-Carbohydrate Diet-Induced Nonalcoholic Fatty Liver Disease by Regulating Nicotinamide Phosphoribosyltransferase/Sirtuin 1 Independent of Carotenoid Cleavage Enzymes in Mice (E01-03)}

\section{Ji Ye Lim, ${ }^{1,3}$ Chun Liu, ${ }^{1}$ Kang-Quan Hu, ${ }^{1}$ Donald E Smith, ${ }^{2}$ and Xiang-Dong Wang ${ }^{1,3}$}

${ }^{1}$ Nutrition and Cancer Biology Lab and ${ }^{2}$ Comparative Biology Unit, Jean Mayer USDA-Human Nutrition Research Center on Aging at Tufts University, MA; and ${ }^{3}$ Biochemical and Molecular Nutrition Program, Friedman School of Nutrition Science and Policy, Tufts University, MA

Background: We have previously reported that a high-refinedcarbohydrate diet (HRCD) is more likely to contribute to nonalcoholic fatty liver disease (NAFLD) than is a high-fat diet with the same caloric content. The HRCD effect is associated with decreased functions of sirtuin 1 (SIRT1). $\beta$-Cryptoxanthin (BCX), a provitamin A carotenoid found primarily in red sweet peppers, has been shown to be protective against NAFLD. BCX can be cleaved by $\beta$-carotene- $15,15^{\prime}$-oxygenase (BCO1), and $\beta$-carotene- $9^{\prime}, 10^{\prime}$-oxygenase (BCO2) to produce bioactive metabolites. $\mathrm{BCO} 1 / \mathrm{BCO} 2$ polymorphisms have been observed in human and rodents.

Objective: In this study, we investigated whether the protective effect of BCX against HRCD-induced NAFLD was dependent on, or independent of, $\mathrm{BCO} 1 / \mathrm{BCO} 2$, and whether upregulation of SIRT1 was the mechanism underlying the physiologic actions of BCX.
Methods: At 6 wk of age, $\mathrm{BCO} 1 / \mathrm{BCO} 2$ double knockout (BCO1/BCO2 DKO) mice and wild-type (WT) mice from a C57BL/6J genetic background were randomly assigned to either the HRCD group $(n=15,66.5 \%$ of energy as carbohydrate including sucrose and maltodextrin) or the HRCD with BCX group ( $n=15,10 \mathrm{mg} \mathrm{BCX} / \mathrm{kg}$ diet) for $24 \mathrm{wk}$. The dose of BCX (paprika extract rich in 11\% BCX from OmniActive) was equivalent to that of daily human consumption of 3-4 oz of a sweet red pepper.

Results: BCX feeding significantly reduced the hepatic steatosis score in both WT and BCO1/BCO2 DKO mice compared with their respective HRCD counterparts $(P<0.05)$. Moreover, BCX feeding significantly reduced hepatic concentrations of triglyceride and total cholesterol levels in BCO1/BCO2 DKO mice compared with the corresponding HRCD group. These hepatic changes caused by BCX feeding were related to the significant upregulation of mRNA and protein levels of nicotinamide phosphoribosyltransferase (NAMPT) and SIRT1, downregulation of mRNA levels of the cholesterol biosynthesis marker (HMG-CoAR) and lipogenesis markers (SREBP-1C, ACC, FAS), and increases in mRNA levels of the fatty acid $\beta$-oxidation marker (PPAR $\alpha$ ).

Conclusion: In conclusion, our results provide compelling experimental evidence that BCX has potentially protective effects against HRCD-induced NAFLD by regulating the NAMPT/SIRT1 pathway independent of carotenoid cleavage enzymes.

\section{Funding Sources}

Supported by NIFA/AFRI (2017-67017-26363) and USDA/ARS (581950-0074).

\section{Nutrition Intervention of Lycopene Ameliorates Atrazine- Induced Neurotoxicity in Mice (E01-04)}

Jin-Long Li, Jia Lin, Xue-Nan Li, Cong Zhang, Jing Ge, Yan-Hua $\mathrm{Li}$

Northeast Agricultural University, China

Background: Widespread contamination by and persistence of residues of the herbicide atrazine (ATR) in the environment results in significant neurotoxicity. Lycopene (LYC), a lipid-soluble antioxidant that has been found to be inversely associated with lipid peroxidation, is considered the most effective antioxidant among the carotenoids. However, the potential of LYC against ATR-induced neurotoxicity remains unknown.

Objective: This study explores the underlying mechanism by which LYC attenuates ATR-induced oxidative damage, the nuclear xenobiotic receptor (NXR) response, cytochrome P450 (CYP450) disorders, and inflammation in the cerebrum.

Methods: To determine the effects of ATR and/or LYC on the brain, mice were treated with ATR (50 or $200 \mathrm{mg} / \mathrm{kg}$ ) and/or LYC (5 mg/kg) by intragastric administration for $21 \mathrm{~d}$. Pathologic alteration of the cerebrum was observed, and the expression of factors related to renal nuclear receptors and CYP450s, oxidative stress, the Nrf2 signaling pathway, and inflammation was detected.

Results: ATR leads to significantly increased histologic alterations. Supplementary LYC significantly decreased ATR-induced dysregulation of oxidative stress markers. LYC treatment downregulated ATRinduced increase of $\mathrm{Nrf} 2$ expression and $\mathrm{Nrf} 2$-regulated detoxification enzymes (NQO-1, HO-1) and antioxidant reductases (SOD1-3, GCLM, 
GCLC, GST, GPx1-8, CAT, TXN1-2, Prx1-6). LYC suppressed ATRinduced activation of NXR (CAR, PXR, AHR, GR, PPAR $\alpha, \operatorname{PPAR} \gamma$, Arnt) and CYP450 disorders. In addition, ATR triggered CYP450 isoform transcription via activation of the cerebral NXR pathway, which can be reversed by LYC. Moreover, ATR-induced upregulation of proinflammatory and downregulation of anti-inflammatory cytokines (upregulation: interleukin 1B and 6, COX2, TRAK6, tumor necrosis factor $\alpha$; downregulation: TLR4, transforming growth factor) and activation of nuclear transcription factor $\kappa \mathrm{B}(\mathrm{NF}-\kappa \mathrm{B})$ mRNA expression.

Conclusion: ATR induced cerebral damage by enhancing oxidative stress and CYP450 disorders, and triggered an inflammatory response by activating the Nrf2/NF- $\kappa$ B signaling pathway. Supplementary LYC significantly alleviated cerebral injury by modulating the Nrf2-mediated antioxidative response and the nuclear receptor-mediated CYP450 response, and by blocking the NF- $\kappa$ B pathway.

\section{Funding Sources}

National Natural Science Foundation of China (31572586), Excellent Youth Foundation of Heilongjiang Province of China (JC2017005), Academic Backbone Project of Northeast Agricultural University (15XG16) and China Agriculture Research System (CARS-35).

Longitudinal Study on Intake of Dietary Lycopene and other Carotenoids in Relation to Gestational Diabetes Mellitus During Mid-Trimester (E01-05)

Qin Gao, ${ }^{1}$ Chunrong Zhong, ${ }^{1}$ Xuezhen Zhou, ${ }^{1}$ Renjuan Chen, ${ }^{1}$ Ting Xiong, ${ }^{1}$ Miao Hong, ${ }^{1}$ Qian Li, ${ }^{1}$ Man Kong, ${ }^{2}$ Weizhen Han, ${ }^{2}$ Guoqiang Sun, ${ }^{3}$ Xuefeng Yang, ${ }^{1}$ Nianhong Yang, ${ }^{1}$ and Liping $\mathrm{Hao}^{1}$

${ }^{1}$ Huazhong University of Science and Technology, China; ${ }^{2}$ The Central Hospital of Wuhan, China; and ${ }^{3}$ Hubei Maternal and Child Health Hospital, China

Background: The antioxidative properties of carotenoids have been shown to reduce the risk of developing type 2 diabetes. However, the relationship between dietary carotenoid intake and gestational diabetes mellitus (GDM) remains unknown.

Objective: We investigated the carotenoid consumption (including $\alpha$-carotene, $\beta$-carotene, $\beta$-cryptoxanthin, lycopene, lutein, and zeaxanthin) of pregnant women during mid-trimester in order to determine whether carotenoid intake affects the prevalence of GDM.

Design: For the present study, the data of 1978 pregnant women recruited for the Tongji Maternal and Child Health Cohort (TMCHC) study, from January 2013 to December 2016, were acquired and analyzed. During mid-trimester, the participants completed an interviewer-administered semiquantitative food-frequency questionnaire to confirm typical dietary carotenoid intake before undertaking the oral glucose tolerance test at 24-28 wk of gestation. Multivariate logistic regression and linear regression were used to obtain the effect estimates.

Results: The median (IQR) intakes of $\alpha$-carotene, $\beta$-carotene, $\beta$-cryptoxanthin, lycopene, lutein, and zeaxanthin were 277.4 (135.1, 649.7) $\mu \mathrm{g}, 3091.4$ (2088.6, 4566.2) $\mu \mathrm{g}, 292.6$ (155.9, 477.4) $\mu \mathrm{g}, 2082.4$ $(145.2,5265.8) \mu \mathrm{g}$, and $2499.4(1571.7,3802.9) \mu \mathrm{g}$, respectively. The multivariable adjusted ORs (95\% CIs) of GDM from the lowest to the highest for lycopene intake were 1.00 (reference), $0.97(0.62,1.51), 0.82$ $(0.51,1.32)$, and $0.50(0.29,0.86)$ ( $P$-trend, 0.007$)$; each SD of logtransformed lycopene consumption increment was associated with a $16 \%(1 \%, 28 \%)$ decrement of GDM $(P=0.042)$. We found that lycopene intake provided significant protection against GDM among primigravid women, as the OR $(95 \% \mathrm{CI})$ of the highest quartile was $0.20(0.07,0.55)$, but not in multiple-pregnancy women $(P$-interaction $=0.036)$. Further multiple linear regression analysis suggested that lycopene intake was inversely related to fasting blood glucose (FBG), rather than to 1 -h postload blood glucose (PBG) and 2-h PBG.

Conclusions: Dietary lycopene intake was inversely associated with GDM risk in mid-pregnancy, especially among primigravid women. Furthermore, the protection was assumed to be due mainly to reduced FBG, instead of to 1-h or 2-h PBG.

\section{Funding Sources}

National Program on Basic Research Project of China (2013FY114200) and National Natural Science Foundation of China (81573149).

Use of a Super-Child Approach and Retinol Isotope Dilution to Estimate Population and Individual Vitamin A Total Body Stores in 3- to 6-Year-Old Mexican Children (E01-06)

Veronica Lopez-Teros, ${ }^{1}$ Jennifer Ford, ${ }^{2}$ Michael H Green, ${ }^{2}$ Sherry A Tanumihardjo, ${ }^{2}$ Brianda Monreal-Barraza, ${ }^{3}$ Lilian Garcia-Miranda, ${ }^{1}$ Bertha I Pacheco-Moreno, ${ }^{3}$ Orlando Tortoledo-Ortiz, ${ }^{3}$ Mauro E Valencia, ${ }^{1}$ and Humberto AstiazaranGarcia $^{3}$

${ }^{1}$ University of Sonora, Mexico; ${ }^{2}$ Pennsylvania State University; and ${ }^{3}$ Research Center for Food and Development, Mexico

Objective: We used compartmental analysis and a super-child approach to estimate both population vitamin A total body stores (VA TBS) and the values for coefficients in a retinol isotope dilution (RID) equation that was then applied to predict TBS in individual children.

Methods: Twenty-four children (3-6 y, mean age $4.8 \pm 0.7 \mathrm{y}$ ) ingested an oral dose of $\left[{ }^{13} \mathrm{C}_{10}\right]$-retinyl acetate $(1 \mathrm{mg})$ in corn oil. Blood (2 samples/child) was collected at 9 time points ( 8 and $12 \mathrm{~h}, 1,2,4,7$, 11,14 and $21 \mathrm{~d}$ post-dose) ( 3 children at a time, except that all subjects were sampled on day 4 ). The geometric mean plasma $\left[{ }^{13} \mathrm{C}_{10}\right]$-retinol fraction of dose versus time data were fit to a previously published 5-compartment model to predict population retinol kinetics and VA TBS, as well as the coefficients at $4 \mathrm{~d}$ needed for the RID equation. Individual TBS was calculated as TBS $=\mathrm{Fa} \times \mathrm{S} \times\left(1 / \mathrm{SA}_{\mathrm{p}}\right)$, where $\mathrm{Fa}$ $\times \mathrm{S}$ is the composite coefficient derived from the model at $4 \mathrm{~d}$, and $\mathrm{SA}_{\mathrm{p}}$ is the plasma retinol specific activity (fraction of dose/ $\mu \mathrm{mol}$ ) at $4 \mathrm{~d}$.

Results: There was no evidence of subclinical inflammation based on C-reactive protein concentration. All children had normal serum retinol concentrations $(1.3 \pm 0.3 \mu \mathrm{mol} / \mathrm{L})$. Retinol kinetics were derived from a 5-compartment model that included two extravascular pools: a faster-turnover pool $(8 \mu \mathrm{mol})$ and a slower turnover pool, presumably vitamin A stores (i.e., TBS; $854 \mu \mathrm{mol}$ ). Taking the population value for $\mathrm{Fa} \times \mathrm{S}$ at $4 \mathrm{~d}(2.3)$ and individual $\mathrm{SA}_{\mathrm{p}}$ on day 4 , the geometric mean VA 
TBS calculated by the RID equation was $837 \mu \mathrm{mol}$ (range: 527-1837 $\mu \mathrm{mol})$.

Conclusion: The model-predicted population VA TBS were similar to the mean of the individual values predicted based on an RID equation at $4 \mathrm{~d}$. Thus, the super-child approach can be used to estimate population VA TBS and coefficients for RID that can be used to calculate the VA TBS in individual children.

\section{Funding Sources}

International Atomic Energy Agency Contract 16880BIM-B; LPG$M$ received a fellowship from the National Research and Technology Council (CONACyT) of Mexico.

Retinal Morphology and Macular Xanthophylls: Implications for Intellectual Ability (E01-07)

Alicia R Jones, Connor Robbs, Caitlyn G Edwards, Anne M Walk, Sharon Thompson, Ginger Reeser, Hannah D Holscher, and Naiman Khan

\section{University of Illinois at Urbana-Champaign}

Objective: The accumulation of macular xanthophyll (i.e., lutein and zeaxanthin) in the retina-measured as macular pigment optical density (MPOD) - is recognized as a biomarker for multiple domains of cognitive function. Additionally, neuroimaging studies have established that retinal structure or morphology corresponds to brain white matter and gray matter, and retinal changes reflect susceptibility to neurodegeneration. However, MPOD and retinal morphometric measures are seldom examined together. Accordingly, this study aimed to delineate the relationships between MPOD and retinal morphometric measures and intellectual ability (IQ) among overweight and obese adults.

Methods: Adults aged 25 -45 y ( $n=53,17$ males) with overweight or obesity [body mass index $\left(\mathrm{kg} / \mathrm{m}^{2}\right): \geq 25.0$ ] underwent optical coherence tomography to assess retinal morphometric measures including total macular (TMV) volume, retinal nerve fiber layer (RNFL) volume, ganglion cell layer (GCL) volume, and central foveal thickness (CFT). MPOD was assessed via customized heteroflicker photometry. Dualenergy X-ray absorptiometry and 7-d diet records were used to assess whole-body adiposity (\%fat), and dietary lutein and zeaxanthin intake (LZ), respectively.

Results: Initial bivariate correlations indicated that IQ was associated with \%fat $(r=-0.25, P=0.04)$ and positively with sex $(r=0.30, P \leq 0.01)$, MPOD $(r=0.26, P=0.03)$, TMV $(r=0.23$, $P \leq 0.05)$, RNFL volume $(r=0.35, P \leq 0.01)$, GCL volume $(r=0.26$, $P=0.03)$, and CFT $(r=0.33, P \leq 0.01)$. However, $\mathrm{LZ}$ intake was not significantly related to IQ $(r=0.20, P=0.16)$. Based on regression modeling, following adjustment for sex and \%fat, only CFT $(\beta=0.28$, $\left.R^{2}=0.17, P=0.03\right)$ and RNFL $\left(\beta=0.33, R^{2}=0.20, P \leq 0.01\right)$ were independent predictors of IQ. MPOD $\left(\beta=0.21, R^{2}=0.14\right.$, $P=0.06)$ and $\operatorname{GCL}\left(\beta=0.23, R^{2}=0.14, P=0.06\right)$ approached statistical significance.

Conclusions: Our data suggest that macular xanthophylls and retinal morphology are markers of intellectual ability in adults. Future work is necessary to continue bridging the gap in the literature relating retinal structural and pigmentation measures to cognitive health.

\section{Funding Sources}

Department of Kinesiology and Community Health at the University of Illinois; the USDA National Institute of Food and Agriculture, Hatch Project (grant 1009249), and the Hass Avocado Board.

Fruit, Vegetables, Carotenoids, and Depression in Puerto Rican Adults (E01-08)

Kaylea Flanagan, Luis Falcon, Katherine L Tucker

University of Massachusetts Lowell

Objective: This research is intended to help better understand the concept of how diet may influence psychological well-being, more specifically, depressive disorder in the Boston Puerto Rican population. Although there are many causes of depression, the major aim of this project was to understand the relationship between fruit and vegetable intake and depressive symptomology. Total plasma carotenoids served as biomarkers for fruit and vegetable intake.

Methods: Data were from the Boston Puerto Rican Health Study (participants at baseline were 45-75 y old). SAS (version 9.4) was used to conduct analyses including univariates, correlations, $t$ tests, and logistic regressions. Depression was measured with the CES-D scale from 0 to 60 . Those with a CES-D score of $\geq 16$ were considered to have depressive symptomology for these analyses. A foodfrequency questionnaire was administered to the population in which they reported their fruit and vegetable intakes, which further included the intake of dietary carotenoids, namely $\alpha$-carotene, $\beta$-carotene, $\beta$-cryptoxanthin, lycopene, and lutein and zeaxanthin. Blood was drawn and total plasma carotenoids were evaluated. After conducting basic analyses, adjustments were made for age, sex, body mass index, smoking status, triglycerides, and total energy intake, as appropriate for each model.

Results: The mean intake of fruit and vegetables was 1.01 servings, with an SD of 0.82 and a range of $0-8.00$. The fruit and vegetable intake variables used were those after an adjustment, which excludes lettuce, potatoes, legumes, and fruit juice. The mean total plasma carotenoid concentration was $92.9 \mu \mathrm{g} / \mathrm{dL}$, with an SD of $38.0 \mu \mathrm{g} / \mathrm{dL}$ and range of $1-399 \mu \mathrm{g} / \mathrm{dL}$. Plasma carotenoid concentration was significantly associated with depressive symptomatology after adjustment for age, sex, and BMI, but this was attenuated in more complex models. Fruit and vegetable intake remained significant across the adjustment for covariates in relation to depressive symptoms.

Conclusion: Fruit and vegetables, mediated by plasma carotenoids, may contribute to improved well-being. On the other hand, those with depression may consume a lower-quality diet. Longitudinal models are needed to clarify the direction of causality.

Funding Sources

NIH P50 HL105185, NIH P01 AG023394, NIH R01AG055948.

Hepatic Stellate Cells Exposed to Astaxanthin During Activation Exhibit a Distinct Metabotype from Quiescent and Activated Hepatic Stellate Cells (P03-001)

Minkyung Bae, Tho Pham, Young-Ki Park, Yoojin Lee, Siqi Hu, Dong-Guk Shin, Pujan Joshi, Seung-Hyun Hong, Nathan Alder, Sung I Koo, and Ji-Young Lee

University of Connecticut 
Objective: Hepatic stellate cells (HSCs) are responsible for the accumulation of excess extracellular matrix, leading to liver fibrosis. We demonstrated that activated HSCs (aHSCs) had higher mitochondrial respiration and lower glycolysis than did quiescent HSCs (qHSCs), and that these changes were attenuated by astaxanthin (ASTX). The objective of this study was to determine the underlying mechanisms by which qHSCs and aHSCs display distinct metabotypes, and how ASTX alters these changes.

Methods: Primary mouse HSCs were cultured on an uncoated plastic dish for activation. The cells at days 1 and 7 served as qHSCs and aHSCs, respectively, and sets of cells were treated with $25 \mu \mathrm{M}$ of ASTX in the presence/absence of FCCP, an uncoupler, during activation. We measured mitochondrial content and cristae structure (by transmission electron microscopy), as well as the expression of genes for mitochondrial biogenesis. The following parameters were also measured to understand the modulation of glycolysis during HSC activation: mRNA levels of genes for glycolysis, such as glucose transporter 1 (Glut1), lactate dehydrogenase A (Ldha), and monocarboxylate transporter 1 (Mct1); phosphorylation of pyruvate dehydrogenase (PDH); and lactate secretion.

Results: aHSCs had higher mitochondrial contents and expression of proliferator-activated receptor $\gamma$ coactivator $1 \beta$ than qHSCs. Inhibition of mitochondrial respiration by FCCP markedly reduced profibrogenic gene expression in aHSCs, which was further reduced by ASTX. ASTX also improved mitochondrial cristae structure with decreased cristae junction width, lumen width, and area. As for glycolysis, aHSCs showed lower mRNA and protein levels of GLUT1, but higher protein levels of phosphorylated PDH than qHSCs, suggesting that aHSCs are likely to have diminished cellular glucose uptake and conversion of pyruvate to acetyl coenzymeA. ASTX increased lactate secretion with concomitant increases in the expression of Ldha and Mct1.

Conclusions: qHSCs showed a glycolytic metabotype, whereas aHSCs had an oxidative metabotype. aHSCs treated with ASTX exhibited a metabotype distinct from qHSCs and untreated aHSCs with increased glycolysis and decreased mitochondrial respiration. Metabotypic changes in HSCs may be a valuable target for the prevention of liver fibrosis.

\section{Funding Sources}

NIH 1R01DK108254-01.

\section{Carotenoids Differentially Regulate Monocyte-Derived Macrophage Phenotype and Functional Polarization (P03-002)}

Xiaoming Gong, ${ }^{1}$ Lewis Rubin, ${ }^{1}$ and Doris Wiener ${ }^{2}$

${ }^{1}$ Texas Tech University Health Sciences Center El Paso; and ${ }^{2}$ Moffitt Cancer Center, FL

Objective: Tumor-associated macrophages (TAMs) are critical stromal components intimately involved in the progression, invasion, and metastasis of cancer cells. In response to microenvironmental signals, monocyte-derived macrophages differentiate into M1-like (classically activated) and M2-like (alternatively activated) macrophages (MФ). Dietary carotenoids are essential components of human nutrition and affect a range of physiologic functions. The objective of this study was to determine the effects of carotene ( $\beta$-carotene, lycopene) and xanthophyll (astaxanthin, lutein) on the differentiation and polarization of human monocyte-derived MФ.

Methods: Monocyte-to-M $\Phi$ differentiation was driven by treatment for $6 \mathrm{~d}$ with granulocyte-macrophage colony-stimulating factor (GM-CSF) or M-CSF \pm individual carotenoids. M1 MФs (+GM-CSF) were activated with lipopolysaccharide + interferon $\gamma$ (IFN $\gamma)$; M2 MФs (+M-CSF) were activated with interleukin (IL) 4.

Results: We validated that M1 and M2 cells showed distinct morphologies and expression patterns (quantitative reverse transcriptasepolymerase chain reaction) of scavenger receptors (SR-A, LDLR, CD36, SR-B1) and cytokines (IL-10, IL-12). M1 and M2 markers (CD80, CD163, CD36, CD14, and CD16) and cytokines (prostaglandin E2, IL-8, IL-6, IL-10, IL-12, tumor necrosis factor $\alpha$, IFN $\gamma$ ) were confirmed by flow cytometry. Expression of CD14, CD16, CD163, CD36, and IL-10 was higher in M2 than in M1 MФs. Lycopene and astaxanthin decreased SRA, CD36, and LDLR mRNA levels in M1 cells but had no significant effect on M2 cells. Lutein also reduced SRA, CD36, and IL-10 expression in M2. Conversely, $\beta$-carotene increased M1 and decreased M2 expression of SRA, CD36, IL-10, and IL-12.

Conclusion: These results show that individual carotenoids can regulate $\mathrm{M} \Phi$ scavenger receptor and cytokine expression. We speculate that dietary carotenoids influence TAM polarization, hence, that modulations in TAMs can greatly affect cancer progression and metastasis.

\section{Funding Sources}

Supported, in part, by grants from the National Institutes of Health (HD42174 and RR18722).

\section{Distribution of an Oral Dose of $\left[{ }^{13} \mathrm{C}\right]-$ Lutein into Tissues of an} Adult Rhesus Macaque: A Pilot Study (P03-003)

Sookyoung Jeon, ${ }^{1}$ Qiyao Li, ${ }_{1}^{1}$ Stanislav Rubakhin, ${ }^{1}$ Jonathan Sweedler, ${ }^{1}$ Josh W Smith, ${ }^{1}$ Martha Neuringer, ${ }^{2}$ Matthew Kuchan, ${ }^{3}$ and John W Erdman $\mathrm{Jr}^{1}$

${ }^{1}$ University of Illinois at Urbana-Champaign; ${ }^{2}$ Oregon Health \& Science University; and ${ }^{3}$ Abbott Nutrition

Objective: Lutein selectively accumulates in the primate retina and brain where it may play an essential role in ocular and cognitive health. To date, no isotopic tracer studies of lutein pharmacokinetics have been performed in a nonhuman primate model. The objective of this pilot study was to assess the distribution of lutein into tissues of an adult rhesus macaque (Macaca mulatta) after a single oral dose of isotopically labeled lutein.

Methods: One 19-y-old female macaque was supplemented daily for a year with $1 \mu \mathrm{mol} \cdot \mathrm{kg}^{-1} \cdot \mathrm{d}^{-1}$ of unlabeled lutein and subsequently provided a single dose of $1.92 \mathrm{mg}$ of $\left[{ }^{13} \mathrm{C}\right]$-lutein $(65 \%$ of lutein was uniformly labeled whereas $35 \%$ remained unlabeled at 1 or 2 carbons). Plasma, 6 brain regions, retina, and other tissues were collected postdose on day 3 . Tissues from an undosed macaque were used as negative controls. Lutein accumulation was quantified by HPLC photodiode array analysis and ${ }^{13} \mathrm{C}$ enrichment was evaluated by LCquadrupole time-of-flight mass spectrometry. 
Results: In the control monkey, $\left[{ }^{12} \mathrm{C}\right]$-lutein, but not $\left[{ }^{13} \mathrm{C}\right]$-lutein, was detectable in serum and all tissues examined. In the ${ }^{13} \mathrm{C}$-dosed macaque, the highest ratio of $\left[{ }^{13} \mathrm{C}\right]$-lutein/ $\left[{ }^{12} \mathrm{C}\right]$-lutein $\left({ }^{13} \mathrm{C} /{ }^{12} \mathrm{C}\right)$ was found in the plasma (11.3\%), followed by the liver (10.6\%), heart (6.9\%), kidney (6.5\%), adrenal gland (3.5\%), and quadriceps (1.5\%). $\left[{ }^{13} \mathrm{C}\right]$-Lutein was detected in the brain and accumulated differentially across regions. The ${ }^{13} \mathrm{C} /{ }^{12} \mathrm{C}$ ratio was highest in the temporal cortex $(2.4 \%)$ and lowest in the subcortical white matter $(0.6 \%)$. $\left[{ }^{13} \mathrm{C}\right]$-Lutein was observed in adipose depots, with the highest ${ }^{13} \mathrm{C} /{ }^{12} \mathrm{C}$ ratio in the axillary brown adipose tissues $(0.8 \%)$ and the lowest in the thigh subcutaneous adipose tissues $(0.1 \%)$. However, $\left[{ }^{13} \mathrm{C}\right]$-lutein was undetectable in macular and peripheral retina, despite high concentrations of $\left[{ }^{12} \mathrm{C}\right]$-lutein.

Conclusions: Three days following dosing, $\left[{ }^{13} \mathrm{C}\right]$-lutein was differentially distributed across various tissues, including multiple brain regions, but was undetectable in the retina. This pilot study demonstrates that distribution of lutein in the macaque is substantially dependent on tissue type. Studies with additional time points are needed to determine if these observations indicate differences in the kinetics of tissue uptake, metabolism, or recycling into circulation.

\section{Funding Sources}

Funding provided by Abbott Nutrition through the Center for Nutrition, Learning and Memory, University of Illinois, UrbanaChampaign and NIH grant P51OD011092.

$\beta$-Carotene Regulates Colon Cancer Stem Cells by Suppressing Cancer Stemness (P03-004)

$\mathrm{Na}$ Youn Lee, ${ }^{1}$ Kyung Eun Lee, ${ }^{1}$ Yoo Sun Kim, ${ }^{1}$ Seung Chul Heo, ${ }^{2}$ and Yuri Kim ${ }^{1}$

${ }^{1}$ Ewha Womans University, Korea; and ${ }^{2}$ Seoul National University Boramae Medical Center, Korea

Objective: Colorectal cancer (CRC), the third most common cancer worldwide, has high rates of recurrence and metastasis. These characteristics are ascribed to cancer stem cells (CSCs), i.e., the small population of cancer cells that possess characteristics of stem cells. $\beta$-Carotene (BC) has been reported to be effective at suppressing CSCs in neuroblastomas. The objective of this study was to analyze the antiCSC effect of BC in colorectal cancer.

Methods: We used clonogenic assays and sphere formation assays to measure the self-renewal capacity of CSCs. CSC markers and the $\mathrm{Wnt} / \beta$-catenin signaling pathway were analyzed in $\mathrm{CD} 133^{+} \mathrm{CD} 44^{+}$ HCT116 and HT29 colon cancer cells, and in primary cells from human CRC tissues. The xenograft model was used to investigate the tumorigenicity of CSC in mice after 10 wk of BC supplementation.

Result: CSCs were isolated after double staining of CD133 and $\mathrm{CD} 44$. BC reduced the number and size of colonies and spheres, indicating that $\mathrm{BC}$ hindered the self-renewal capacity of CSCs. BC also downregulated CSC markers, including CD44, CD133, ALDH1A1, NOTCH1, and Sox 2 , and the Wnt $/ \beta$-catenin signaling pathway, including $\beta$-catenin in $\mathrm{CD} 133^{+} \mathrm{CD} 44^{+} \mathrm{HCT} 116$ and HT-29 cells. The antiCSC effect of BC was confirmed in primary cells isolated from human CRCs. In addition, the number and size of tumors were decreased and the time of onset of tumor was delayed by $\mathrm{BC}$ supplementation in xenograft mice injected with $\mathrm{CD} 133^{+} \mathrm{CD} 44^{+} \mathrm{HCT} 116$ cells. BC also inhibited CSC markers and the $\mathrm{Wnt} / \beta$-catenin signaling pathway in tumors in vivo.

Conclusions: BC exerted an inhibitory effect on colon CSCs by regulating cancer stemness, indicating its therapeutic potential for the treatment of CRC.

\section{Funding Sources}

Supported by the Basic Science Research Program through the National Research Foundation of Korea (NRF) funded by the Ministry of Education (NRF-2016R1D1A1B03932018) and Brain Korea 21 Plus (project no. 22A20130012143).

Ablation of Carotenoid Cleavage Enzymes (BCO1 and BCO2) Induces Nonalcoholic Fatty Liver Disease by Altering the Farnesoid $\mathrm{X}$ Receptor/microRNA-34a/Sirtuin 1 Pathway in Mice (P03-005)

Ji Ye Lim, ${ }^{1,3}$ Chun Liu, ${ }^{1}$ Kang-Quan Hu, ${ }^{1}$ Donald E Smith, ${ }^{2}$ Xiang-Dong Wang ${ }^{1,3}$

${ }^{1}$ Nutrition and Cancer Biology Lab and ${ }^{2}$ Comparative Biology Unit, Jean Mayer USDA-Human Nutrition Research Center on Aging at Tufts University, MA; and ${ }^{3}$ Biochemical and Molecular Nutrition Program, Friedman School of Nutrition Science and Policy, Tufts University, MA

Background: Nonalcoholic fatty liver disease (NAFLD) is the most commonly occurring chronic liver disease worldwide. Both genetic and environmental factors are related to susceptibility to NAFLD. $\beta$-Carotene-15,15' -oxygenase (BCO1) and $\beta$-carotene- $9^{\prime}, 10^{\prime}$ oxygenase $(\mathrm{BCO} 2)$ are essential enzymes in carotenoid metabolism. Although $\mathrm{BCO} 1 / \mathrm{BCO} 2$ polymorphisms have been associated with alterations to human and animal carotenoid levels, recent studies have suggested that $\mathrm{BCO} 1$ or $\mathrm{BCO} 2$ may have specific functions beyond the cleavage of carotenoids.

Objective: In the present study, we investigated the effect of ablation of both $\mathrm{BCO} 1 / \mathrm{BCO} 2$ on the development of NAFLD and its underlying molecular mechanism(s).

Methods: Wild-type mice (WT, $n=8$ ) and BCO1/BCO2 double knockout mice (BCO1/BCO2 DKO, $n=8$ ) with a $\mathrm{C} 57 \mathrm{BL} / 6 \mathrm{~J}$ genetic background were fed a standard rodent chow diet for $24 \mathrm{wk}$.

Results: There were no changes in body weight or liver weights between WT and BCO1/BCO2 DKO groups. However, all BCO1/BCO2 DKO mice developed liver steatosis ( 8 of 8 mice) and had significantly higher levels of hepatic triglyceride and total cholesterol levels in the liver and plasma compared with WT mice ( 0 of 8 mice, $P<0.05)$. These hepatic changes in the $\mathrm{BCO} 1 / \mathrm{BCO} 2 \mathrm{DKO}$ mice were associated with the following significant effects: 1) increases in mRNA and protein levels of lipogenesis markers (SCD1, ACC, CD36), and decreases in the gene expressions of fatty acid $\beta$-oxidation markers (CPT- 1 , SIRT3, PPAR $\alpha$ ); 2) upregulation of the cholesterol metabolism markers (CYP7A1, CYP8B1, HMG-CoAR); 3) alterations to the microRNAs (miR-34a, miR-33, miR-122) related to triglyceride accumulation and cholesterol metabolism; 4) increases in hepatic oxidative stress markers (HO-1, SOD1, SOD2, GPX, catalase); and 5) decreases in nuclear protein levels of farnesoid X receptor (FXR), and mRNA levels of FXR, small heterodimer partner (SHP), and sirtuin 1 (SIRT1). 
Conclusion: Taken together, the present study provided novel experimental evidence that the ablation of both $\mathrm{BCO} 1 / \mathrm{BCO} 2$ led to the development of $\mathrm{NAFLD}$, indicating that $\mathrm{BCO} 1 / \mathrm{BCO} 2$ could play a significant role in maintaining normal hepatic lipid and cholesterol homeostasis, potentially through the activation of the $\mathrm{FXR} / \mathrm{miR}$ 34a/SIRT1 pathway.

\section{Funding Sources}

Supported by NIFA/AFRI (2017-67,017-26,363) and USDA/ARS (58-1950-0074).

Dietary Lycopene Feeding Inhibits Cigarette Smoke-Induced Chronic Obstructive Pulmonary Disease and Lung Preneoplastic Lesions in a Ferret Model (P03-006)

Jelena Mustra Rakic, ${ }^{1}$ Chun Liu, ${ }^{2}$ Sudipta Veeramachaneni, ${ }^{2}$ Lynne Ausman, ${ }^{3}$ and Xiang-Dong Wang ${ }^{2,3}$

${ }^{1}$ Tufts University, MA; ${ }^{2}$ Jean Mayer USDA-Human Nutrition Research Center on Aging at Tufts University, MA; and ${ }^{3}$ Friedman School of Nutrition Science and Policy, Tufts University, MA

Background: Lung cancer and chronic obstructive pulmonary disease (COPD) are caused by cigarette smoke (CS). 4(Methylnitrosamino)-1-(3-pyridyl)-1-butanone (NNK) is the most potent carcinogen in CS. Epidemiologic studies suggest an inverse association between the consumption of lycopene, a major carotenoid naturally occurring in tomatoes and tomato products, and the risk of lung cancer.

Objective: We hypothesize that lycopene protects against CS/NNK-induced COPD and preneoplastic lesions in the ferret lung.

Methods: Ferrets, a nonrodent model that closely mimics human carotenoid accumulation and lung lesions, were randomly divided into four groups ( $n=12-16 /$ group): control, NNK + CS, NNK + CS + low lycopene (LL, $2.2 \mathrm{mg} \cdot \mathrm{kg}$ body $\mathrm{wt}^{-1} \cdot \mathrm{d}^{-1}$ ) and $\mathrm{NNK}+\mathrm{CS}+$ high lycopene $\left(\mathrm{HL}, 6.6 \mathrm{mg} \cdot \mathrm{kg}\right.$ body $\left.\mathrm{wt}^{-1} \cdot \mathrm{d}^{-1}\right)$. Ferrets in the NNK $+\mathrm{CS}$ groups were given NNK (50 mg/kg body wt, i.p. injection) once a month for 4 consecutive months, and were exposed to CS for $15 \mathrm{~min} / \mathrm{d}$ over a 22 -wk period. Ferrets in the lycopene feeding groups were fed with lycopene 3 wk prior to NNK injection at 2 doses, LL and HL, which are equivalent to $\sim 30$ and $\sim 90 \mathrm{mg}$ lycopene/d in humans, respectively, and continued feeding over a 22 -wk period. Lycopene concentrations were measured by HPLC. Bronchitis was evaluated by histopathology defined by peribronchial/peribronchiolar/alveolar infiltrates of inflammatory cells. The degree of emphysema, i.e., the alveolar destruction, was assessed by the measurement of the mean linear intercept (Lm). Preneoplastic lesions, including squamous metaplasia and atypical adenomatous hyperplasia, were evaluated by histopathology.

Results: We did not observe a significant difference in body weights among groups. There was a higher accumulation of lycopene in the HL group than in the LL group in both plasma and liver. Both lycopene doses significantly inhibited CS/NNK-induced emphysema development, as compared with the NNK + CS alone $(P<0.05)$. $\mathrm{HL}$ feeding significantly inhibited bronchitis in the CS/NNK-exposed group $(P<0.05)$, whereas LL feeding showed marginal significance $(P=0.09)$. Additionally, HL feeding significantly decreased CS/NNKinduced preneoplastic lesion development $(P<0.05)$.
Conclusions: These data provide strong supportive evidence that lycopene has a beneficial effect against COPD and pulmonary preneoplastic lesions.

Funding Sources

Supported by NIH/NCI (CA176256) and USDA/ARS (58-19500074S).

Reducing Carotenoid Degradation Is Key to Maintaining Nutritional Quality of High Carotenoid Biofortified Maize During Storage (P03-007)

Smith G Nkhata, ${ }^{1}$ Darwin Ortiz, ${ }^{1}$ Dieudonne Baributsa, ${ }^{1}$ Bruce

Hamaker, ${ }^{1}$ Torbert Rocheford, ${ }^{1}$ and Mario G Ferruzzi ${ }^{2}$

${ }^{1}$ Purdue University, IN; and ${ }^{2}$ North Carolina State University

Objective: Provitamin A carotenoid (PVAC)-rich biofortified maize holds promise as a means to address vitamin A deficiency in developing countries. However, carotenoid degradation during postharvest storage can significantly reduce PVAC content. In this study, we assessed the use of the Purdue Improved Crop Storage (PICS) bag to slow oxidative degradation of carotenoids. PICS bags are a lowcost grain storage system that provide a hermetically sealed, oxygendeprived environment for control of pests. We hypothesized that maize stored in oxygen-reduced PICS bags would show reduced carotenoid loss and be of higher nutritional quality.

Methods: Freshly harvested grain from 2 biofortified maize genotypes, OPVI and OPVII, was dried $(\sim 8.5 \%$ moisture $)$ and stored for 8 mo in PICS-oxy (oxygen scavenging), PICS-noxy (no scavenging) and woven bags each at $50 \mathrm{~kg} / \mathrm{bag}$ at $\sim 29^{\circ} \mathrm{C}$ and $\sim 30 \%$ relative humidity. Carotenoid content was determined by LC with diode array detection at $0,2,4$, and $8 \mathrm{mo}$, and percentage of recovery was calculated by comparing individual and total carotenoid (TC) contents at each time point with the initial content.

Results: Initial carotenoid contents of OPVI compared with OPVII were 37.0 and 22.7 (zeaxanthin), 7.2 and 11.4 (lutein), 2.8 and 2.7 ( $\beta$-cryptoxanthin), 2.5 and 3.2 (trans- $\beta$-carotene), and 3.5 and 3.9 (cis$\beta$-carotene) $\mu \mathrm{g} / \mathrm{g}$ dry weight. When comparing the PICS-oxy, PICSnoxy, and woven bags after $4 \mathrm{mo}$, OPVI retained significantly higher levels of PVAC than OPVII (76\%, 51\%, 56\% compared with 57\%, $47 \%, 46 \% ; P<0.05)$, and TC retention in OPVI was significantly higher than in OPVII $(90 \%, 73 \%, 71 \%$ compared with $76 \%, 69 \%$, $64 \%)$. PICS-oxy had higher retention than PICS-noxy and woven bags, indicating that both genotype and storage system had a significant effect on carotenoid stability and confirming entrapped oxygen and oxidation as a primary mechanism of carotenoid degradation in postharvest storage. After 8 mo, retention of PVAC was similar but higher in OPVI $(45 \%, 44 \%, 38 \%)$ compared with OPVII $(34 \%, 34 \%$, $34 \%)$. TC retention for OPVI $(63 \%, 48 \%, 51 \%)$ was higher than for OPVII $(51 \%, 49 \%, 43 \%)$ in PICS-oxy, PICS-noxy, and woven bags, respectively.

Conclusions: The stability of carotenoids during post-harvest storage is dependent on low oxygen content, genotype, and storage period. Furthermore, the PICS storage system can be an effective method to enhance the post-harvest stability of carotenoids.

Funding Sources

USAID BHEARD Scholarship. 